\title{
Erros relacionados à medicação nas Unidades de Terapia Intensiva: revisão integrativa
}

\author{
Errors related to medication in Intensive Care Units: integrative review \\ Errores relacionados con la medicación en las Unidades de Cuidados Intensivos: revisión \\ integrativa
}

Susany Franciely Pimenta ${ }^{1 *}$, Carolina Rodrigues Milhorini ${ }^{2}$, Luiz Fernando da Silva ${ }^{2}$.

\section{RESUMO}

Objetivo: Analisar as produções cientificas acerca dos principais erros de medicação na Unidade de Terapia Intensiva (UTI), assim como as principais estratégias que visem a segurança do paciente. Métodos: revisão integrativa, com busca nas bases de dados PubMed, MEDLINE, LILACS, BDENF e SciELO, por artigos científicos publicados no período de Janeiro de 2014 a Janeiro de 2019. Foram utilizados os descritores: Erros de medicação, Unidades de Terapia Intensiva e Segurança do paciente. Resultados: Obteve-se uma amostra de 10 artigos que adequam com o tema e enquadraram-se nos critérios. A maior parte dos estudos analisados foram realizados no exterior, assim revelando a necessidade de maior estímulo à pesquisa e publicação no Brasil, voltadas aos erros de medicação na (UTI), uma vez que é considerado um setor frequente para essas ocorrências.Conclusão: Concluiu-se que são necessárias intervenções no processo de medicar, a fim de garantir a prestação de uma assistência mais segura nas Unidades de Terapia Intensiva.

Palavras-chave: Erros de medicação, Unidades de terapia intensiva, Segurança do paciente.

\begin{abstract}
Objective: to analyze the scientific productions about the main medication errors in the Intensive Care Unit (ICU), as well as the main strategies aimed at patient safety. Methods: integrative review, with search in the PubMed, MEDLINE, LILACS, BDENF and SciELO databases, for scientific articles published from January 2014 to January 2019. The following descriptors were used: Medication errors, Intensive Care Units and Patient safety. Results: We obtained a sample of 10 articles that fit the theme and fit the criteria. Most of the studies analyzed were performed abroad, thus revealing the need for a greater stimulus to research and publication in Brazil, focused on medication errors in the Intensive Care Unit, since it is considered a frequent sector for these occurrences. Conclusion: It was concluded that interventions in the medication process are necessary in order to guarantee the provision of safer care in the Intensive Care Units.
\end{abstract}

Keywords: Medication errors, Intensive care units, Pacient safety.

\section{RESUMEN}

Objetivo: analizar las producciones científicas sobre los principales errores de medicación en la Unidad de Cuidados Intensivos (UCl), así como las principales estrategias dirigidas a la seguridad del paciente. Métodos: revisión integradora, para buscar en las bases de datos PubMed, MEDLINE, LILACS, SciELO y BDENF para artículos científicos publicados entre enero de 2014 y enero de 2019. Los descriptores se utilizaron: Los errores de medicación, unidades de cuidados intensivos y Seguridad del paciente. Resultados:

1Faculdade Pitágoras, Londrina- Paraná. ^E-mail: susanypimenta@hotmail.com

¿Universidade Estadual de Londrina (UEL), Londrina- Paraná.

SUBMETIDO EM: 05/2019

ACEITO EM: 06/2019

PUBLICADO EM: 07/2019

REAS/EJCH | Vol. Sup.27 | e931 | DOI: https://doi.org/10.25248/reas.e931.2019 Página 1 de 6 
Se obtuvo una muestra de 10 artículos que adecuan al tema y se encuadraron en los criterios. La mayor parte de los estudios analizados se realizaron en el exterior, revelando así la necesidad de un mayor estímulo a la investigación y publicación en Brasil, dirigidas a los errores de medicación en la Unidad de Cuidado Intensivo, una vez que se considera un sector frecuente para esas ocurrencias.Conclusión: Se concluyó que son necesarias intervenciones en el proceso de medicar, a fin de garantizar la prestación de una asistencia más segura en las Unidades de Cuidados Intensivos.

Palabras clave: Errores de medicación; Unidades de cuidados intensivos; Seguridad del paciente.

\section{INTRODUÇÃO}

Apesar dos medicamentos serem imprescindíveis para a sobrevida de um paciente crítico, o uso indevido gera erros que podem acarretar agravos à saúde do paciente, bem como transtornos econômicos à instituição de saúde. Os erros são definidos como falhas associadas ao uso inadequado de um ou mais fármacos, podendo acarretar qualquer danos ao cliente. A responsabilidade do erro é multiprofissional, uma vez que os erros podem acontecer em qualquer etapa do processo da terapia medicamentosa, que são: o armazenamento, prescrição, dispensação, preparação e administração do medicamento (GUZZO GM, et al., 2018; COSTA MP, et al., 2018; NUTE C, 2014; SANTOS DM, et al., 2014; CARVALHO ML, et al., 2013).

Supõe-se que, nas Unidades de Terapia Intensiva (UTI), esses incidentes atinjam 947 pessoas a cada mil, por dia no mundo, sendo gastos até 2,8 milhões de dólares com o tratamento das complicações. Estima-se que na América mais de $30 \%$ das medicações realizadas resultaram em erros de medicação, ocasionando em milhares de incapacitades por ano. Tais incidentes vêm sendo configurado como um problema mundial na saúde pública e privada (AZEVEDO FFM, et al., 2015; SHEHATA ZHA, et al., 2015).

A Unidade de Terapia Intensiva (UTI) é considerada o setor o mais vulnerável aos erros relacionados a medicação, uma vez que o estado de saúde dos pacientes é crítico, necessitando maiores demandas do profissional frente a tantas medicações por longos períodos. A falta de profissionais e protocolos para auxiliálos, bem como as péssimas condições de trabalho culminam em erros de medicação, muitas vezes irreversíveis (FERREIRA PC, et al., 2014; MURILLO PMA, et al ., 2016; AZEVEDO FILHO FM, et al., 2015; RODRIGUEZ EOL, et al., 2017; SOUZA RF, et al., 2018).

Sem dúvida, ações que garantam a segurança do paciente é primordial ao sistema de saúde, uma vez que, além de evitarem gastos desnecessários, alimentam a excelência da assistência prestada ao cliente (SILVA JSD, et al., 2017; DUARTE SCM, et al., 2016).

Diante disso, esse estudo objetiva identificar nas produções científicas acerca dos principais erros de medicações na Unidade de Terapia Intensiva (UTI), assim como as principais estratégias de prevenção que visem a segurança do paciente.

\section{MÉTODOS}

O presente estudo trata-se de uma revisão integrativa a qual buscou responder quais são os principais erros de medicação na Unidade de Terapia Intensiva (UTI) e as estratégias que visem a segurança do paciente.

A busca dos periódicos foi realizada na Medical Literature Analysis and Retrieval System Online (MEDLINE), Literatura Latino-Americana e do Caribe em Ciências da Saúde (LILACS), Base de Dados de Enfermagem (BDENF), National Library of Medicine (PubMed) e Scientific Electronic Library Online (SciELO), utilizando os descritores "Erros de medicação", "Unidade de Terapia Intensiva" e "Segurança do paciente", selecionados através do DeCS (Descritores em Ciência da Saúde) e MESH (Medical Subject Headings) para indexar na busca em português e inglês, respectivamente. 
A seleção dos artigos obedeceu aos seguintes critérios de inclusão: todos os artigos que versavam com o tema, livres ou privados, publicados entre Janeiro de 2014 a Janeiro de 2019, utilizando o operador Boleano "AND" para realizar as associações e sem limitação de idiomas.

Após o levantamento bibliográfico, realizou-se a leitura rigorosa dos títulos e resumos dos artigos para selecionar aqueles que condiziam com os critérios de inclusão supracitados. Nos casos em que o títulos e o resumos não foram suficientes para definir a temática pesquisada, buscou-se a publicação na íntegra, de forma que todos os critérios pudessem ser aplicados a fim de analisar sua relevância e suas respectivas contribuições úteis para integrar as informações, assim como as estratégias de prevenção.

\section{RESULTADOS}

Foram encontrados um total de 172 artigos, dos quais, dez versavam sobre o tema. Destes, quatro eram publicações nacionais e seis publicações internacionais. Ao analisar os estudos encontrados, identificou-se que a maioria foi publicado em 2015, seguido de 2018 (Tabela 1).

Com relação ao cenário dos estudos, nove foram realizados em unidade de terapia intensiva e apenas um foi realizado em diversos setores hospitalares incluindo a UTI. Grande parte dos estudos não definiram se os hospitais utilizados como local de estudo eram públicos ou privados maioria na realizada com pacientes adultos.

Tabela 1 - Caracterização dos artigos que compuseram o estudo.

\begin{tabular}{|c|c|c|c|c|}
\hline Local & Título do artigo & Periódico & Ano & $\begin{array}{l}\text { População de } \\
\text { estudo }\end{array}$ \\
\hline SciELO & $\begin{array}{l}\text { Assistência segura ao paciente no preparo e } \\
\text { administração de medicamentos }\end{array}$ & $\begin{array}{l}\text { Revista Gaúcha } \\
\text { de Enfermagem }\end{array}$ & 2018 & Adulto \\
\hline BDENF & $\begin{array}{l}\text { Vivência de enfermeiras(os) acerca dos } \\
\text { incidentes relacionados à administração de } \\
\text { medicamentos em terapia intensiva }\end{array}$ & $\begin{array}{l}\text { JOURNAL OF } \\
\text { NURSING AND } \\
\text { HEALTH }\end{array}$ & 2018 & Adulto \\
\hline LILACS & $\begin{array}{l}\text { Segurança da terapia medicamentosa em } \\
\text { neonatologia: olhar da enfermagem na } \\
\text { perspectiva do pensamento ecológico } \\
\text { restaurativo }\end{array}$ & $\begin{array}{l}\text { Texto \& Contexto- } \\
\text { Enfermagem }\end{array}$ & 2018 & Neonato \\
\hline PubMed & $\begin{array}{l}\text { Facilitated Nurse Medication-Related Event } \\
\text { Reporting to Improve Medication Management } \\
\text { Quality and Safety in Intensive Care Units }\end{array}$ & Nursing research & 2017 & Adulto/Pediátrico \\
\hline PubMed & $\begin{array}{l}\text { Impact of the introduction of a specialist critical } \\
\text { care pharmacist on the level of pharmaceutical } \\
\text { care provided to the critical care unit }\end{array}$ & $\begin{array}{l}\text { International } \\
\text { Journal of } \\
\text { Pharmacy } \\
\text { Practice }\end{array}$ & 2016 & Adulto \\
\hline PubMed & $\begin{array}{l}\text { Pharmacist's review and outcomes: } \\
\text { Treatment-enhancing contributions tallied, } \\
\text { evaluated, and documented (PROTECTED- } \\
\text { UK) }\end{array}$ & $\begin{array}{l}\text { Journal of critical } \\
\text { care }\end{array}$ & 2015 & Adulto \\
\hline PubMed & $\begin{array}{l}\text { Los errores de tratamiento en una unidad } \\
\text { neonatal,uno de los principales } \\
\text { acontecimientos adversos }\end{array}$ & $\begin{array}{l}\text { Anales de } \\
\text { Pediatría }\end{array}$ & 2015 & Neonato \\
\hline SciELO & $\begin{array}{l}\text { Prevalência de incidentes relacionados à } \\
\text { medicação em unidade de terapia intensiva }\end{array}$ & $\begin{array}{l}\text { Acta Paulista de } \\
\text { Enfermagem }\end{array}$ & 2015 & Adulto \\
\hline PubMed & $\begin{array}{l}\text { Descriptive analysis of medication errors } \\
\text { reported to the Egyptian national online } \\
\text { reporting system during six months }\end{array}$ & $\begin{array}{l}\text { Journal of the } \\
\text { American Medical } \\
\text { Informatics } \\
\text { Association }\end{array}$ & 2015 & Adulto \\
\hline MEDLINE & Reducing medication errors & $\begin{array}{l}\text { Art \& science } \\
\text { medicines } \\
\text { management }\end{array}$ & 2014 & Adulto \\
\hline
\end{tabular}

Fonte: Produção do próprio autor, 2019. 
Os estudos foram conduzidos principalmente no que diz respeito a assistência prestada aos clientes no preparo e administração de medicamentos, fundamentado que os erros poderiam ser prevenidos, visto que a enfermagem participa no final do processo.

\section{DISCUSSÃO}

Os medicamentos são essenciais para o tratamento e controle das complicações relacionadas a saúde, promovendo a melhoria na qualidade de vida do indivíduo. Entretanto, o uso inadequado pode comprometer a eficácia desejada, além de causar danos à saúde do paciente, que variam desde reações alérgicas, incapacidade e morte. Isto também é permeado por impacto econômico, uma vez que as consequências prolongam o tempo de internação e custos no ambiente hospitalar que poderiam ser investidos em outras necessidades (AZEVEDO FFM, et al., 2015; RODRIGUEZ EOL, et al., 2017, COSTA MP, 2018).

A literatura aponta que a quantidade de medicamentos prescritos nas Unidades de Terapia Intensiva (UTI) são duas vezes maiores do que outras unidades e que a incidência de erros também ultrapassa qualquer setor. Isto se deve ao grau de complexidade dos pacientes que necessitam de diversas medicações por um longo período e que qualquer anormalidade gera grandes intercorrências clinicas, ressaltando a importância do controle dessa prática (AZEVEDO FFM, et al., 2015, COSTA MP, et al., 2018; RODRIGUEZ EOL, et al., 2017; XU J, et al., 2018; RICHTER A, et al., 2016).

Cada profissional é responsável pela prevenção na ocorrência de possíveis erros de medicação. $O$ processo é multiprofissional, cabendo ao médico a responsabilidade pela avaliação do paciente e prescrição, ao farmacêutico a reavaliação da prescrição médica e dispensação do medicamento e à enfermagem a preparação, administração e monitoramento, não cabendo apenas à última a represália do advento (SHULMAN R, et al., 2015; NUTE C, 2014; GUZZO GM, et al., 2018).

Entretanto, a equipe de enfermagem tem o importante papel de buscar e identificar possíveis falhas neste processo, caracterizando-se como uma barreira fundamental para interditar eventos adversos relacionados à fármacos. Isto se deve ao fato de que este profissional estará no fim da cadeia do processo de medicação, tendo a oportunidade de levantar questionamentos e impedir qualquer equívoco (GUZZO GM, et al., 2018; COSTA MP, et al., 2018).

Para que seja possível este empenho das equipes, readequações essenciais para a diminuição dos erros relacionados a medicação a níveis aceitáveis nas UTIs são imprescindíveis. Cabe às instituições desenvolverem e implementarem protocolos de fácil acesso, com informações científicas atualizadas e principalmente relacionados a segurança do paciente no ambiente hospitalar, incluindo informações sobre o uso de novos fármacos e treinamentos contínuos para equipe multiprofissional, abordando todas as etapas da medicação e a readequação no quadro de funcionários (COSTA MP, et al., 2018; GUZZO GM, et al., 2018; RUIZ ME, et al., 2015; SHEHATA ZHA, et al., 2015).

Outro fator contribuinte para os erros de medicação são as prescrições médicas, pois a desinformação dessa classe acerca da terapêutica mais indicada, ilegibilidade das prescrições e múltiplas abreviações não padronizadas implicam diretamente no trabalho da enfermagem, ocorrendo atrasos no processo e comprometendo a segurança do paciente. A implantação da prescrição eletrônica e fiscalização dessa prática compõe uma das formas mais eficazes de diminuir os erros de medicação relacionadas a esta barreira. Outra tática que pode vetar o erro proveniente da desinformação das drogas é a inclusão de um farmacêutico clínico na equipe, buscando evitar equívocos e interações medicamentosas (RODRIGUEZ EOL, et al., 2017; NUTE C, 2014; RICHTER A, et al., 2016; SHULMAN R, et al.,2015).

A Unidade de Terapia Intensiva nem sempre propicia um local de trabalho adequado para a preparação e administração da medicação. Há uma grande sobrecarga de trabalho e interrupções constantes, que resultam em distrações, estresse e cansaço na equipe de enfermagem. Organizar um local específico para preparo de medicação, sem que haja telefones, prontuários ou qualquer outro paralelo assegura concentração e fidedignidade ao processo, facilitando sua checagem. Respeitar o número máximo de pacientes por 
profissional é crucial, devendo a instituição ousar de bom senso nas contratações. (XU J, et al., 2018; RODRIGUEZ EOL, et al., 2017; SHEHATA ZHA, et al., 2015; AZEVEDO FFM, et al., 2015; RUIZ ME, et al., 2015; COSTA MP, et al., 2018; NUTE C, 2014).

Erros graves também ocorrem pela deficiência na identificação do paciente, principalmente em casos onde este apresenta rebaixamento do nível de consciência, por dois motivos: inutilização de pulseiras e placas de identificação do paciente no leito; falha de conferência do nome descrito no prontuário com o da prescrição e subsequentemente com o nome descrito na pulseira de identificação. A literatura sugere que questionar o nome completo do paciente e manter, ao menos, dois identificadores em locais visíveis à beira leito funcionam como estratégias assertivas em relação ao paciente correto. Vale salientar que esta protege o paciente do erro independentemente da faixa etária e do nível de consciência deste (RODRIGUEZ EOL, et al., 2017).

Os erros de medicação intensificam-se no setor de UTI neonatal. A problemática é justificada pela fragilidade dos prematuros, o que demanda maiores intervenções e internações prolongadas para a completa reabilitação. Devido à falta de experiência da equipe multiprofissional com essa clientela e aos medicamentos não serem direcionados para cada faixa etária infantil, aumenta-se a inadequabilidade na dosagem prescrita e nos cálculos de diluição e rediluição. As complicações desses erros variam de acordo com a concentração e classe medicamentosa, porém nessa população um pequeno erro gera uma avalanche de subsequentes que ameaçam a continuidade da vida dos neonatos. (RUIZ ME, et al., 2015; GUZZO GM, et al., 2018).

Identificar as causas dos erros de medicação é indispensável para um funcionamento adequado da instituição, todavia esta, na maioria das vezes, não se configura como um tarefa fácil. Uma barreira frequente para essa ação é o medo de represália que os profissionais têm em assumir um desacerto, portanto a melhor forma de obter dados concretos da incidência de erros no setor e ter a possibilidade de intervir nos pontos principais é instituir uma política anônima que rompa punições e qualquer julgamento, uma vez que, como apontado anteriormente, não há apenas um culpado (AZEVEDO FFM et al., 2015; COSTA MP, et al., 2018; GUZZO GM, et al., 2018; RUIZ ME, et al., 2015; SHEHATA ZHA, et al., 2015; NUTE C , 2014).

A Organização Mundial de Saúde (OMS) está constantemente em busca de desenvolver estratégias afim de melhorar a qualidade da assistência e garantir a segurança do paciente, portanto, para que as estratégias sejam eficazes faz-se necessário que as instituições de saúde invistam em recursos tecnológicos, científicos e estruturais (RODRIGUEZ EOL, et al., 2017).

\section{CONCLUSÃO}

Constata-se que os erros de medicação são uma realidade frequente nas unidades de terapia intensiva, sendo necessário identificar e compreender quais são os fatores que resultam em erros de medicação e a frequência com que ocorrem, a fim de programar estratégias que os reduzam e previnam. Vale ressaltar a necessidade de intervenção institucional para que haja redução da incidência. Acentua-se a importância e necessidade de desenvolvimento de novas pesquisas acerca do tema para que haja uma visão mais detalhada em cenários diferentes, bem como a formulação e implementação de estratégias que possam melhorar ainda mais a realidade das Unidades de Terapia Intensiva ao redor do Brasil e do mundo, diminuindo morte e agravos evitáveis. A presente revisão não foi norteada por conflitos de interesse.

\section{REFERÊNCIAS}

1. AZEVEDO FFM, et al. Prevalência de incidentes relacionados à medicação em unidade de terapia intensiva. Acta Paulista de Enfermagem, 2015; 28(4): 331-336.

2. CARVALHO ML, et al. Strategies for the prevention of errors in medication administration: a contribution to nursing practice. Revista de Pesquisa: Cuidado é Fundamental Online, 2013; 5(6): 390-400.

3. COSTA MP, et al. Vivência de enfermeiras (os) acerca dos incidentes relacionados à administração de medicamentos em terapia intensiva. J. nurs. health, 2018; 8(2): e188207.

4. DUARTE SCM, et al. Error characterization in intensive care nursing. Cogitare Enferm, 2016; 21: 1-8.

REAS/EJCH | Vol. Sup.27 | e931 | DOI: https://doi.org/10.25248/reas.e931.2019 Página 5 de 6 
5. FERREIRA PC, et al. Adverse event versus medication error: perceptions of nursing staff acting in intensive care. Revista de Pesquisa: Cuidado é Fundamental Online, 2014; 6 (2): 725-734.

6. GUZZO GM, et al. Segurança da terapia medicamentosa em neonatologia: olhar da enfermagem na perspectiva do pensamento ecológico restaurativo. Texto \& contexto enfermagem, 2018; 27(3): e4500016.

7. MURILLO PMA, et al. Análisis del registro de un sistema de notificación de incidentes en una unidad de cuidados críticos. Enfermería Intensiva, 2016; 27(3): 112-119.

8. NUTE C. Reducing medication erros. Nursing Standard, 2014; 29(12): 45-51.

9. RICHTER A, et.al. Impact of the introduction of a specialist critical care pharmacist on the level of pharmaceutical care provided to the critical care unit. International Journal of Pharmacy Practice, 2016; 24(4): 253-261.

10. RODRIGUEZ EOL, et al. Assistência segura ao paciente no preparo e administração de medicamentos. Revista Gaúcha de Enfermagem, 2017; 38 (4): e2017-0029.

11. RUIZ ME, et al. Los errores de tratamiento en una unidad neonatal, uno de los principales acontecimientos adversos. Anales de Pediatría, 2016; 84(7): 211-217.

12. SANTOS DM, et al. Segurança do paciente: fatores causais de eventos adversos a medicamentos pela equipe de enfermagem, Caderno de Graduação-Ciências Biológicas e da Saúde, 2014; 2(2): 19-30.

13. SHEHATA ZHA, et al. Descriptive analysis of medication errors reported to the Egyptian national online reporting system during six months. Journal of the American Medical Informatics Association, 2015; 23(2): 366-374.

14. SHULMAN R, et al. Pharmacist's review and outcomes: Treatment-enhancing contributions tallied, evaluated, and documented (PROTECTED-UK). Journal of critical care, 2015; 30(4): 808-813.

15. SILVA JSD, et al. Erros de prescrição e administração envolvendo um medicamento potencialmente perigoso. Rev. enferm. UFPE online, 2017; 11(10): 3707-717.

16. SOUZA RF, et al. Eventos adversos na Unidade de Terapia Intensiva. Rev. enferm. UFPE online, 2018; 12(1): 1927.

17. XU J, et al. Facilitated Nurse Medication-Related Event Reporting to Improve Medication Management Quality and Safety in Intensive Care Units. Nursing research, 2017; 66(5): 337-349. 